Proceedings of the International Symposium on Physics of Materials (ISPMA 14), September 10-15, 2017, Prague

\title{
Defects in High Entropy Alloy HfNbTaTiZr Prepared by High Pressure Torsion
}

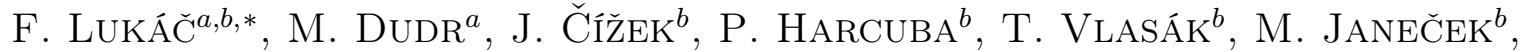 \\ J. KURIPLACH ${ }^{b}$, J. MOON ${ }^{c}$, H.S. KIM ${ }^{c}$, J. ZÝKA ${ }^{d}$ AND J. MÁLEK ${ }^{d}$ \\ ${ }^{a}$ Institute of Plasma Physics CAS, Za Slovankou 3, 182 00, Praha, Czech Republic \\ ${ }^{b}$ Faculty of Mathematics and Physics, Charles University, V Holesovickach 2, 18000 Praha 8, Czech Republic \\ ${ }^{c}$ Department of Materials Science and Engineering, POSTECH, Pohang 790-784 \\ ${ }^{d}$ UJP PRAHA a.s., Nad Kamínkou 1345, 156 00, Prague, Czech Republic
}

\begin{abstract}
High entropy alloy HfNbTaTiZr was successfully processed by severe plastic deformation using high pressure torsion (HPT) and ultrafine grained microstructure was achieved. The microstructure of HPT-deformed HfNbTaTiZr alloy was characterized by X-ray diffraction and compared with conventionally cast ingots. The lattice defects introduced by HPT processing were characterized by positron annihilation spectroscopy. The X-ray diffraction profiles of HTP-deformed samples were extremely broadened due to small sizes of coherently diffracting domains and a high microstrain introduced by severe plastic deformation.
\end{abstract}

DOI: 10.12693/APhysPolA.134.891

PACS/topics: 78.70.Bj, 61.72.Ff

\section{Introduction}

High entropy alloys exhibit various combinations of interesting physical properties due to the formation of solid solution stabilized by high configuration entropy. Refractory metal high entropy alloy HfNbTaTiZr exhibits single phase solid solution with bcc structure $[1,2]$. The alloy has high yield strength $\left(\sigma_{0.2}=929 \mathrm{MPa}\right.$ in compression [1] and $\sigma_{0.2}=1155 \mathrm{MPa}$ in tension [2]). Structure refinement may improve the strength and ductility of HfNbTaTiZr alloy as was already demonstrated by conventional cold rolling [3]. Methods of severe plastic deformation (SPD) enable a strong grain refinement down to nanoscale [4-7] and may, therefore, further improve mechanical properties of HfNbTaTiZr alloy. HPT is the most efficient technique for structure refinement by severe plastic deformation $[5,6]$. In the present work HPT was employed for preparation of ultrafine grained (UFG) HfNbTaTiZr alloy. Severe plastic deformation does not only cause grain refinement but also introduces a high density of lattice defects (dislocations and vacancies) [8]. HPT straining introduces the lateral inhomogeneity of strain with the minimum in the centre of the disk and continuous increase towards its periphery and results in the inhomogeneous microstructure distribution of coarse grains in the centre and fine grains near the disk periphery. The microstructure inhomogeneity is continuously smeared out with increasing strain (number of HPT revolutions) $[5,6]$. Hence the structure refinement starts at the margin of the sample disk and gradually extends towards the centre and relatively homogeneous ultrafine

*corresponding author; e-mail: lukac@ipp.cas.cz grained structure across the sample is usually achieved after sufficient number of HPT revolutions [5]. In the present work the development of microstructure during HPT processing of HfNbTaTiZr alloy was investigated using electron microscopy combined with X-ray line profile analysis and positron annihilation spectroscopy.

\section{Experimental}

Ingots of HfNbTaTiZr alloy were prepared by vacuum arc melting from pure metals of $99.9 \%$ purity placed in water cooled copper crucible. Casting was performed eight times and the ingot was flipped between each two melts to mix the elements properly and suppress any chemical heterogeneity [2]. Thin discs of diameter $20 \mathrm{~mm}$ and thickness of $1 \mathrm{~mm}$ were cut from cast ingot and processed by high pressure torsion (HPT) under the pressure of $2.5 \mathrm{GPa}$ for different numbers of HPT revolutions $N=1 / 4,1 / 2,1,5,15$. The sample only pressed by the high pressure without any torsion straining $(N=0)$ has been prepared as well.

Microstructure of the material before and after deformation was investigated using electron backscatter diffraction (EBSD) technique. The sample surfaces were polished with argon ion polisher. Zeiss Auriga Compact scanning electron microscope (SEM) operated at $10 \mathrm{keV}$ equipped with EDAX EBSD camera was used.

Phase composition was measured by X-ray diffraction on a D8 Discover (Bruker, DE) diffractometer equipped with 1D detector LynxEye and $\mathrm{Cu} K_{\alpha}$ line beam anode. The X-ray diffraction studies have been performed in the Bragg-Brentano symmetrical geometry with $20 \mathrm{~mm}$ wide divergent beam. The whole diffraction profile was fitted by means of Rietveld refinement of XRD peaks in TOPAS \# v5 software [9]. Correction of peak intensities of powder diffraction patterns for preferred orientation was treated by the March-Dollase approach [10]. The 
size of coherently diffracting domains (CDD) and the microstrain were determined from broadening of Lorentzian and Gaussian components of pseudo-Voigt profile function, respectively.

Positron annihilation spectroscopy (PAS) was performed using a ${ }^{22} \mathrm{Na}$ positron source with activity of 1.0 MBq deposited on a $2 \mu \mathrm{m}$ thick mylar foil. A digital spectrometer described in [11] was employed for positron lifetime (LT) measurements. The spectrometer was equipped with $\mathrm{BaF}_{2}$ scintillators and fast photomultipliers Hamamatsu H3378. Detector pulses were sampled using a couple of 8-bit ultrafast digitizers Acqiris DC 211 (Agilent Technologies) with the sampling frequency of $4 \mathrm{GHz}$. The spectrometer exhibits an excellent time resolution of 145 ps (full width at half maximum of the resolution function). At least $10^{7}$ positron annihilation events were collected in each LT spectrum. The source contribution to the LT spectra consisted of two components with lifetimes of 368 ps and 1.5 ns and corresponding relative intensities 11 and $1 \%$ representing contributions of positrons annihilating in the ${ }^{22} \mathrm{Na}$ source spot and the covering mylar foil, respectively.

\section{Results and discussion}

The EBSD map of as-cast HfNbTaTiZr alloy is shown in Fig. 1a. The as-cast sample exhibits the mean grain size around $300 \mu \mathrm{m}$. The EBSD map of the only pressed sample $(N=0)$ is shown in Fig. 1b. One can see in the figure that compression by high pressure without torsion straining did not lead to any significant grain refinement. The EBSD map of the sample subjected to HPT straining applying $N=1 / 2 \mathrm{HPT}$ revolution is shown in Fig. 1c. The EBSD map was measured in the centre of the sample disc corresponding to the rotation axis. Substantial structure refinement caused by HPT processing is evident in Fig. 1c. However, the structure of the HPT-deformed sample is not homogeneous after $1 / 2 \mathrm{HPT}$ rotation. One can see in Fig. 1c that the sample $N=1 / 2$ consists of regions with UFG structure co-existing with coarse grains containing deformation twins. Hence, plastic deformation of HfNbTaTiZr alloy during HPT processing occurs by the slip of dislocations and also by twining. Further HPT straining led to so high degree of imposed strain that EBSD indexing was impossible.

Figure 2 shows X-ray diffraction patterns for the ascast sample and HPT-deformed samples subjected to various numbers $N$ of $\mathrm{HPT}$ revolutions. The as-cast sample contains single bcc phase with the lattice parameter of $a=3.4059(1) \AA$ which is consistent with the value reported by Senkov et al. [1]. Samples subjected to HPT deformation exhibit extremely broadened diffraction peaks. Moreover, the texture change has been observed in the samples subjected to HPT straining.
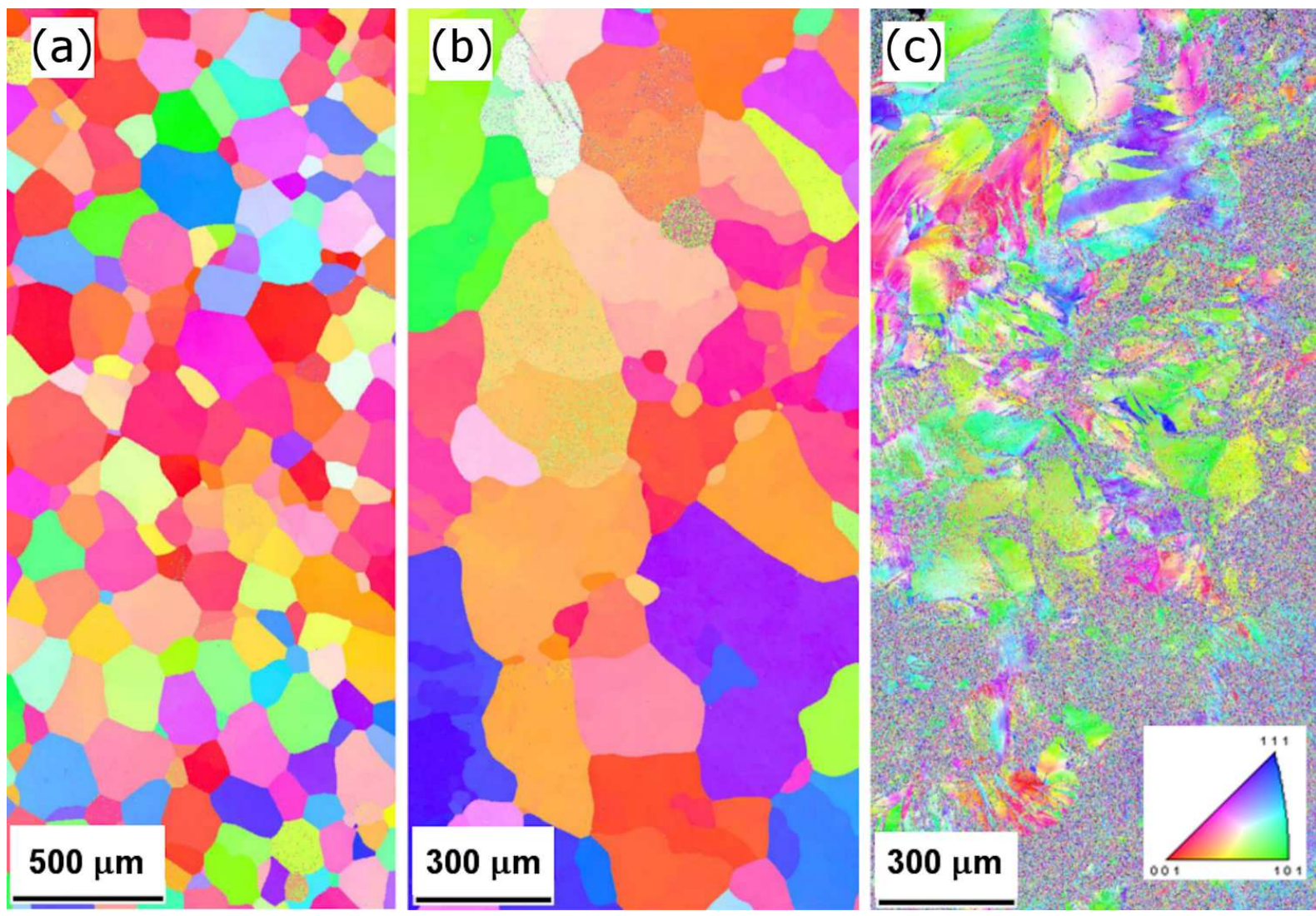

Fig. 1. EBSD maps of HfNbTaTiZr alloy (a) as-cast sample (b) sample compresed by high pressure of 2.5 GPa without any torsion deformation (number of HPT revolutions $N=0$ ) (c) HPT-defomed sample $N=1 / 2$. The EBSD map was measured in the centre of the HPT-deformed sample. 
March-Dollase [10] treatment of texture in powder diffraction method includes a correction of peak intensities since the angle between the preferred orientation vector and the diffraction vector may change. Therefore, two preferred orientations of grains are determined independently, which alter the intensity of all model diffraction peaks. In the as-cast and only pressed sample $(N=0)$ the texture components (110) and (200) dominate, while in samples deformed by HPT straining the dominating texture components are (110) and (211) already after $1 / 4$ of rotation.

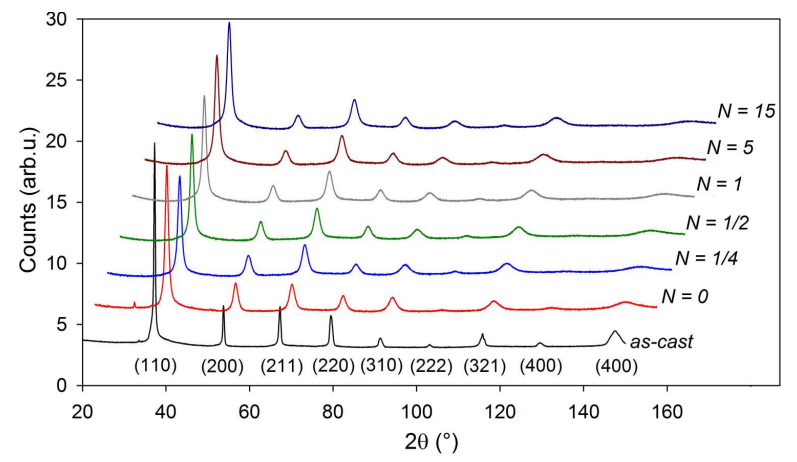

Fig. 2. The development of X-ray diffraction patterns for HfNbTaTiZr alloy during HPT straining for various number of HPT revolutions $N$.

Figure 3 shows results of the Rietveld refinement fitting of XRD diffraction patterns of HfNbTaTiZr samples. The lattice parameter slightly increases in the early stages of HPT processing. The size of CDD substantially decreases after deformation and saturates for $N \geq 1$. Moreover, the microstrain $e_{0}$ induced by deformation increases with HPT straining and reaches a saturated value in order of $3.5 \times 10^{-3}$. Note that unusually high defect concentration was reported in HfNbTaTiZr alloy deformed by compression using plastic strain values between 2 and 20\% [12], i.e. subjected to significantly lower deformation compared to HPT. Similarly, a high dislocation density in the order of $10^{16} \mathrm{~m}^{-2}$ was determined by X-ray line profile analysis in HPT-deformed $\mathrm{CrMnFeCoNi}$ high-entropy alloy [12].

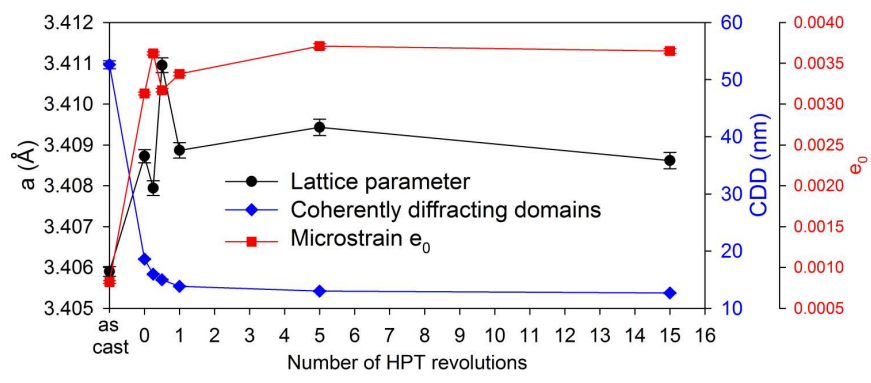

Fig. 3. Results of the Rietveld refinement fits for HfNbTaTiZr alloy.

Figure 4 shows the mean positron lifetime measured for all samples studied. The as-cast sample exhibits the mean positron lifetime of $159(1)$ ps. The as-cast sample may contain defects introduced during casting and shaping. In order to recover these defects the as-cast sample was annealed at $1000^{\circ} \mathrm{C}$ for $2 \mathrm{~h}$ sealed in evacuated quartz ampoule. After annealing the surface of the sample was chemically etched to remove a thin oxide layer formed during annealing. The mean positron lifetime of the annealed sample is 146(1) ps. This value is close to the bulk lifetime of HfNbTaTiZr alloy obtained by by our preliminary $a b$-initio theoretical calculations. Hence, the density of defects in the annealed sample is very low and almost all positrons are annihilated in the free state. On the other hand, the as-cast sample contains defects introduced by cutting and polishing and the mean positron lifetime is increased by the presence of these open volume defects.

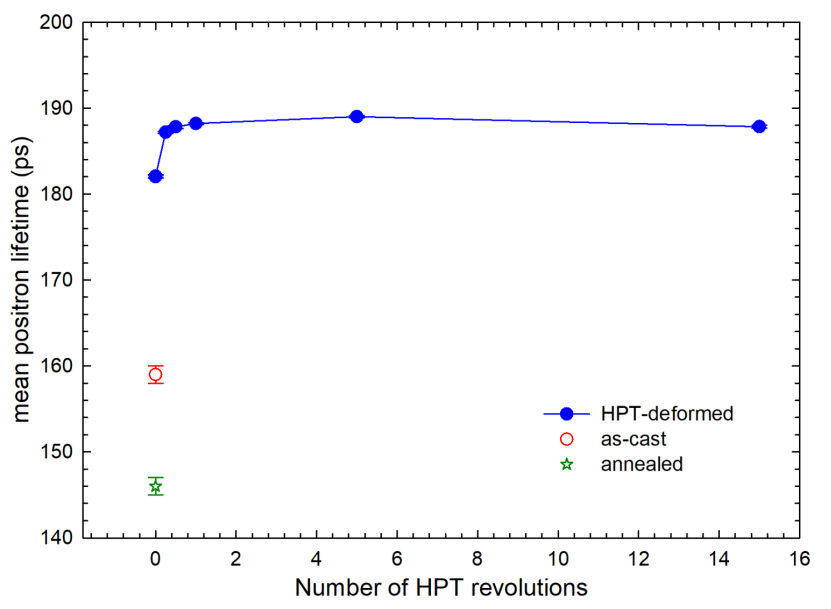

Fig. 4. The mean positron lifetime for HfNbTaTiZr alloy subjected to HPT straining. The PAS measurements were always performed in the centre of the sample disc.

The mean positron lifetime for the only pressed sample $(N=0)$ is remarkably higher than that for the ascast sample testifying that even compression by a high pressure introduced a high density of defects. The HPT straining led to further increase in the concentration of defects which is reflected by an additional increase of the mean positron lifetime. For $N \geq 1$ the mean positron lifetime saturates at value around 189 ps since the density of defects becomes so high that virtually all positrons are trapped at defects (saturated trapping). Since severe plastic deformation introduces dislocations as dominating type of defects and vacancies are created by nonconservative motion of dislocations the observed positron lifetime of 189 ps can be attributed to vacancy-like defects. Note that dislocation line itself is only a shallow positron trap and positron trapping in dislocations is a two step process where positron is firstly weakly localized in the dislocation line and such pre-trapped positron rapidly diffuses along the dislocation line until it is eventually trapped at a vacancy bound to the dislocation [13]. Hence the final positron annihilation site is a vacancy squeezed by the compressive elastic field of dislocation 
and the lifetime of positrons trapped at dislocations is, therefore, slightly shorter than that for plain vacancies. One can expect that the positron lifetime of 189 ps is just the mean value of certain distribution of positron lifetimes trapped in vacancies with open volume varying due to variations in the proportion of the chemical elements (Hf, Nb, Ta, Ti, Zr) surrounding the vacancy. Since vacancies are surrounded by elements with various atomic radius, the lattice relaxations around vacancies change with fluctuations in the local chemical composition. This causes fluctuations in the open volume of vacancies reflected by variations of the lifetime of trapped positrons.

\section{Conclusions}

The structure of refractory metal high entropy alloy HfNbTaTiZr was successfully refined by HPT. The Rietveld refinement of X-ray diffraction patterns showed that the mean size of coherently scattering domains strongly decreases during HPT straining while the microstrain increases due to lattice defects created by severe plastic deformation. This was confirmed by positron annihilation spectroscopy. The mean positron lifetime exhibits a pronounced increase with HPT straining and reaches a saturated value when single HPT revolution is completed. Similarly, the mean size of coherently scattering domains and the microstrain become saturated after single HPT revolution.

\section{Acknowledgments}

This work was supported by the Czech Science Foundation (project 17-17016S). P.H. and M.J. acknowledge financial support by ERDF under the project CZ.02.1.01/0.0/0.0/15 003/0000485. J.M. and H.S.K. acknowledge financial support by the Future Material Discovery Project of the National Research Foundation of Korea (NRF) funded by the Ministry of Science and ICT of Korea (NRF-2016M3D1A1023383).

\section{References}

[1] O.N. Senkov, J.M. Scott, S.V. Senkova, D.B. Miracle, C.F. Woodward, J. Alloys Compd. 509, 6043 (2011).

[2] J. Zýka, J. Málek, Z. Pala, I. Andršová, J. Vesely, in: METAL 2015 - 24th International Conference on Metallurgy and Materials, Brno 2015, p. 1687.

[3] O.N. Senkov, S.L. Semiatin, J. Alloys Compd. 649, 1110 (2015)

[4] P.W. Bridgman, Phys. Rev. 48, 825 (1935).

[5] A. Zhilyaev, T. Langdon, Prog. Mater. Sci. 53, 893 (2008).

[6] R.Z. Valiev, R.K. Islamgaliev, I.V. Alexandrov, Prog. Mater. Sci. 45, 103 (2000).

[7] F. Lukáč, J. Čížek, J. Knapp, I. Procházka, P. Zháňal, R.K. Islamgaliev, J. Phys. Conf. Ser. 674, 012007 (2016).

[8] J. Čížek, M. Janeček, O. Srba, R. Kužel, Z. Barnovská, I. Procházka, S. Dobatkin, Acta Mater. 59, 2322 (2011).

[9] A.A. Coelho, TOPAS, Coelho Software, Brisbane 2016.

[10] W.A. Dollase, J. Appl. Crystallogr., 19, 267, (1986).

[11] F. Bečvář, J. Čížek, I. Procházka, J. Janotová, Nucl. Instrum. Methods Phys. Res. A 539, 372 (2005).

[12] G. Dirras, J. Gubicza, A. Heczel, L. Lilensten, J.-P. Couzinié, L. Perričre, I. Guillot, A. Hocini, Mater. Charact. 108, 1 (2015).

[13] L.C. Smedskjaer, M. Manninen, M.J. Fluss, J. Phys. F Met. Phys. 10, 2237 (1980). 